\title{
On a Relationship Between the Convergents of the Nearest Integer and Regular Continued Fractions
}

\author{
By William W. Adams
}

Abstract. In this paper we derive a relation concerning the speed of convergence of the nearest integer and regular continued fractions. If $A_{n} / B_{n}, p_{k} / q_{k}$ denote the convergents of the nearest integer and regular continued fractions of an irrational number $\alpha$, then for all $n$ there is a $k(n)$ such that $A_{n} / B_{n}=p_{k(n)} / q_{k(n)}$. It is shown that

$$
\lim _{n \rightarrow \infty} \frac{n}{k(n)}=\frac{\log \left(\frac{1+\sqrt{5}}{2}\right)}{\log 2}
$$

for almost all $\alpha$. This problem is reduced to a special case of a general result concerning the frequency of partial quotients in the regular continued fraction (Theorem 2).

1. Introduction. Williams and Buhr [7] compared the lengths of the algorithms for computing the fundamental unit of real quadratic fields using the regular continued fraction* (RCF) and the nearest integer continued fraction (NICF) (see Section 4 for the definition of the NICF). It is known (see [5], say, for RCF and [6], [7], [8] for NICF) that, for square-free positive integers $D$, if $a+b \sqrt{D}$ is a fundamental unit of $Q(\sqrt{D})(a, b$ positive integers or halves of positive integers if $D \equiv 1(\bmod 4))$, then $a / b$ is a convergent of both the RCF and N!ICF. Let us suppose that the fundamental unit is given by the $p(D)$ th convergent of the RCF and the $\pi(D)$ th convergent of the NICF. It is observed in [7] that the ratio $\pi(D) / p(D)$ is near $\log 2=.693147181 \ldots$. Indeed, in an example of D. Shanks [4] of a prime $D=26437680473689$ with an unusually long period for its RCF, it is observed that $\pi(D) / p(D)=.6942215829 \ldots$. Moreover, it is noted that

$$
\frac{\sum_{\substack{d \leqslant D \\ d \text { squarefree }}} \pi(d)}{\sum_{\substack{d \leqslant D \\ d \text { squarefree }}} p(d)}=.7017174 \ldots
$$

for $D=100000$.

The purpose of this paper is to derive what the asymptotic value of these ratios should be on the assumption that, within the period of $\sqrt{D}$, the RCF behaves like

Received November 15, 1978.

AMS (MOS) subject classifications (1970). Primary 10K10, $10 \mathrm{~K} 15$.

*The RCF is called the ordinary continued fraction (OCF) in [7]. 
almost all numbers with respect to the frequency of certain partial quotients. It will be shown that, with this assumption, the above ratios will be, as $D \rightarrow \infty$,

$$
\frac{\log \left(\frac{1+\sqrt{5}}{2}\right)}{\log 2}=.6942419 \ldots .
$$

This agrees with Shanks' example to four places. As Shanks pointed out, this exceptional accuracy was really to be expected since the frequencies of the small partial quotients of the RCF, as reported in [4] , agree with the Gauss-Kuzmin law to that same accuracy.

Now let $\alpha$ be any irrational number. Denote by $p_{k} / q_{k}$, the convergents of the RCF $\left(p_{k}=p_{k}(\alpha)\right)$, and by $A_{n} / B_{n}$, the convergents of the NICF. So $p_{k}, q_{k}$ are relatively prime positive integers with $q_{0}<q_{1}<q_{2}<\cdots$, and $A_{n}, B_{n}$ are relatively prime integers such that $\left|B_{0}\right|<\left|B_{1}\right|<\left|B_{2}\right|<\cdots$. As we shall see below, each $A_{n} / B_{n}$ is one of the $p_{k} / q_{k}$. So there is a strictly increasing function $k(n)$ such that for all $n \geqslant 0$

$$
A_{n} / B_{n}=p_{k(n)} / q_{k(n)} .
$$

We will show the following theorem.

THEOREM 1. For almost all real numbers $\alpha$

$$
\lim _{n \rightarrow \infty} \frac{n}{k(n)}=\frac{\log \left(\frac{1+\sqrt{5}}{2}\right)}{\log 2} .
$$

This result will be deduced from the following general metrical theorem concerning the RCF. For irrational $\alpha$ with $0<\alpha<1$ let

$$
\alpha=\left[0 ; a_{1}(\alpha), a_{2}(\alpha), \ldots\right]
$$

denote its RCF. Set $\alpha_{j}=\left[a_{j}(\alpha) ; a_{j+1}(\alpha), \ldots\right]$.

THEOREM 2. Let $\alpha, \beta$ be irrational numbers with $0<\alpha, \beta<1$.

(a) Define, for all integers $\nu \geqslant 0, \psi_{\nu}(n, \alpha, \beta)$ to be the number of integers $j$ satisfying $0 \leqslant j \leqslant n-1$

(1) $a_{j+1}(\alpha)=a_{1}(\beta), \quad a_{j+2}(\alpha)=a_{2}(\beta), \ldots, a_{j+\nu}(\alpha)=a_{\nu}(\beta), \quad \alpha_{\nu+j+1}>a_{\nu+1}(\beta)$.

Then, for almost all $\alpha$,

$$
\lim _{n \rightarrow \infty} \frac{1}{n} \sum_{\nu=0}^{\infty}(-1)^{\nu} \psi_{\nu}(n, \alpha, \beta)=\frac{\log (1+\beta)}{\log 2} .
$$

(b) Define, for all integers $\nu \geqslant 0, \varphi_{\nu}(n, \alpha, \beta)$ to be the number of integers $j$ satisfying $0 \leqslant j \leqslant n-\nu$ and (1). Then for almost all $\alpha$

$$
\lim _{n \rightarrow \infty} \frac{1}{n} \sum_{\nu=0}^{\infty}(-1)^{\nu} \varphi_{\nu}(n, \alpha, \beta)=\frac{\log (1+\beta)}{\log 2} \text {. }
$$


We note that if

$$
\beta=\frac{\sqrt{5}-1}{2}=[0 ; 1,1, \ldots],
$$

then the last condition in (1) is automatic. Thus $\psi_{\nu}$ and $\varphi_{\nu}$, in this case, are simply counting the number of sequences of $\nu 1$ 's in a row.

The relevance of the frequency of occurrence of the partial quotients is seen in

THEOREM 3. For a given integer $n \geqslant-1$, suppose

$$
\frac{A_{n}}{B_{n}}=\frac{p_{k}}{q_{k}} .
$$

Then

$$
\frac{A_{n+1}}{B_{n+1}}=\frac{p_{k+1}}{q_{k+2}} \text { or } \frac{p_{k+2}}{q_{k+2}},
$$

where the latter occurs if and only if $a_{k+2}=1\left(a_{k+2}=a_{k+2}(\alpha)\right)$. Moreover,

$$
\frac{A_{-1}}{B_{-1}}=\frac{p_{-1}}{q_{-1}} .
$$

Actually, the relevant frequencies for Theorem 1 are the frequencies of the sequences $1 ; 1,1 ; 1,1,1 ; \ldots$. This accounts for the appearance of the number $(1+\sqrt{5}) / 2$ in Theorem 1 .

Now suppose that $\alpha=\sqrt{D}$, and suppose that $A_{n} / B_{n}$ gives the fundamental unit of $Q(\sqrt{D})$. Then, of course, $p_{k(n)} / q_{k(n)}$ also gives the fundamental unit, and so $\pi(D)$ $=n$ and $p(D)=k(n)$. Thus, on the assumption that as $D$ gets large, the first $p(D)$ partial quotients behave like almost all numbers with respect to the sequences of digits $1 ; 1,1 ; 1,1,1$; etc., I conjecture that

$$
\lim _{\substack{D \rightarrow \infty \\ D \text { squarefree }}} \frac{\pi(D)}{p(D)}=\frac{\log \left(\frac{1+\sqrt{5}}{2}\right)}{\log 2} .
$$

I am indebted to H. C. Williams for allowing me the use of his manuscripts [7], [8] in advance of publication. The present manuscript was prepared before [8] was available to me, but it should be pointed out that Theorem 3 was already contained in [8].

I would also like to take this opportunity to thank D. Shanks for his encouragement during the course of the preparation of this paper.

Finally, as this paper was being typed, the author discovered that G. J. Rieger [9] has proved a result which yields an analogous result to Theorem 1 for the average length of RCF and NICF for rational numbers. 
2. Proof of Theorem 2(a). The purpose of this section is to prove Theorem 2(a). We will follow the Ergodic Theorem proof for results of this nature (see [1, pp. 40-50]). For any $v \geqslant 0$ define a function $f_{\nu}\left(u_{1}, \ldots, u_{\nu}, x\right)$, for positive integers $u_{1}, \ldots, u_{\nu}$ and real numbers $x>1$, to be 1 provided $u_{j}=a_{j}(\beta)(1 \leqslant j \leqslant \nu)$ and $x>$ $a_{\nu+1}(\beta)$ and to be zero otherwise. Define the transformation $T$ of the unit interval $0<\alpha<1$ by

$$
T(\alpha)=\frac{1}{\alpha}-\left[\frac{1}{\alpha}\right]
$$

where $[y]$ denotes the largest integer $\leqslant y$. If $T^{j}$ denotes $T$ composed with itself $j$ times, we have

$$
\psi_{\nu}(n, \alpha, \beta)=\sum_{j=0}^{n-1} f_{\nu}\left(a_{1}\left(T^{j} \alpha\right), \ldots, a_{\nu}\left(T^{j} \alpha\right), T^{j} \alpha_{\nu+1}\right)
$$

Set

$$
f(\alpha)=\sum_{\nu=0}^{\infty}(-1)^{\nu} f_{\nu}\left(a_{1}(\alpha), \ldots, a_{\nu}(\alpha), \alpha_{\nu+1}\right) .
$$

We note that if $T^{j} \alpha \neq \beta$ for all $j \geqslant 0$ (in particular if $\alpha$ is not equivalent to $\beta$ ), then this sum for $f\left(T^{j} \alpha\right)$ is in fact finite $(j \geqslant 0)$. There are only a countable number of such $\alpha$ and so $f\left(T^{j} \alpha\right)$ is defined for almost all $\alpha$ in $(0,1)$, for all $j \geqslant 0$. Then, for $\alpha$ not equivalent to $\beta$,

$$
\lim _{n \rightarrow \infty} \frac{1}{n} \sum_{\nu=0}^{\infty}(-1)^{\nu} \psi_{\nu}(n, \alpha, \beta)=\lim _{n \rightarrow \infty} \frac{1}{n} \sum_{j=0}^{n-1} f\left(T^{j} \alpha\right)
$$

(the interchange of summations in (2) is justified since the sums are actually finite).

Now define the Gauss measure $\mu$ on $(0,1)$ by

$$
\mu(E)=\frac{1}{\log 2} \int_{E} \frac{d x}{1+x},
$$

for Lebesgue measurable sets $E$ in $(0,1)$. It is well known, [1, p. 44] that $T$ preserves $\mu$ and is ergodic with respect to $\mu$. Then, if we knew that $f \in L_{1}(\mu)$, we would have from the Ergodic Theorem [1, p. 13], and (2) that

$$
\lim _{n \rightarrow \infty} \frac{1}{n} \sum_{\nu=0}^{\infty}(-1)^{\nu} \psi_{\nu}(n, \alpha, \beta)=\int_{0}^{1} f(x) d \mu(x)
$$

for almost all $x$ in $(0,1)$. This will now be verified and at the same time the integral will be evaluated.

Set

$$
\varphi^{*}(\nu)=\int_{0}^{1} f_{\nu}\left(a_{1}(x), \ldots, a_{\nu}(x), x_{\nu+1}\right) d \mu(x)
$$

It is not hard to show (see [2, Chapter III]) that $f_{\nu}\left(a_{1}(x), \ldots, a_{\nu}(x), x_{\nu+1}\right)$ is the characteristic function of the set of $x$ in interval with endpoints 


$$
\frac{p_{\nu}(\beta)}{q_{\nu}(\beta)}=\frac{p_{\nu}}{q_{\nu}}, \quad \frac{p_{\nu+1}(\beta)}{q_{\nu+1}(\beta)}=\frac{p_{\nu+1}}{q_{\nu+1}}
$$

Hence,

$$
\begin{aligned}
\varphi^{*}(\nu) & =\frac{1}{\log 2}\left|\log \left(1+\frac{p_{\nu}}{q_{\nu}}\right)-\log \left(1+\frac{p_{\nu+1}}{q_{\nu+1}}\right)\right| \\
& \leqslant \frac{1}{\log 2}\left|\frac{p_{\nu}}{q_{\nu}}-\frac{p_{\nu+1}}{q_{\nu+1}}\right|=\frac{1}{\log 2} \frac{1}{q_{\nu} q_{\nu+1}}
\end{aligned}
$$

Thus,

$$
\sum_{\nu=0}^{\infty} \varphi^{*}(\nu)<\infty
$$

(it is well known that $q_{\nu} \geqslant 2^{(\nu-1) / 2}$ ). Hence by Fubini's theorem, $f \in L_{1}(\mu)$ and, moreover, (using Fubini's theorem again and (4))

$$
\begin{aligned}
\int_{0}^{1} f(x) d \mu(x) & =\sum_{\nu=0}^{\infty}(-1)^{\nu} \varphi^{*}(\nu) \\
& =-\frac{1}{\log 2} \sum_{\nu=0}^{\infty}\left(\log \left(1+\frac{p_{\nu}}{q_{\nu}}\right)-\log \left(1+\frac{p_{\nu+1}}{q_{\nu+1}}\right)\right) \\
& =\frac{\log (1+\beta)}{\log 2}
\end{aligned}
$$

(since $p_{0} / q_{0}=0$ and $p_{\nu} / q_{\nu} \rightarrow \beta(\nu \rightarrow \infty)$ and $\left.p_{\nu} q_{\nu+1}-p_{\nu+1} q_{\nu}=(-1)^{\nu+1}\right)$. This completes the proof of Theorem 2(a).

Using the techniques of [3], it should be possible to derive an explicit error term in Theorem 2(a) and, hence, using the result of the next section, an error term in Theorem 2(b), also.

3. Proof of Theorem 2(b). Define

$$
\rho_{\nu}(n, \alpha, \beta)=\psi_{\nu}(n, \alpha, \beta)-\varphi_{\nu}(n, \alpha, \beta) .
$$

It suffices to show that, except on a set of measure zero in $\alpha$, we have

$$
\lim _{n \rightarrow \infty} \frac{1}{n} \sum_{\nu=0}^{\infty} \rho_{\nu}(n, \alpha, \beta)=0 .
$$

Now $\rho_{\nu}(n, \alpha, \beta)$ is the number of integers $j, n-\nu+1 \leqslant j \leqslant n-1$ satisfying (1). Denote by $E_{\nu, n, j}$ the set of $\alpha$ satisfying (1). We first estimate the measure $\left|E_{\nu, n, j}\right|$ of $E_{\nu, n, j}$

We follow the argument given in [2, pp. 57-60]. If $\alpha \in E_{\nu, n, j}$, then for some positive integers $b_{1}, \ldots, b_{j}$,

$$
\alpha=\left[0 ; b_{1}, b_{2}, \ldots, b_{j}, a_{1}(\beta), \ldots, a_{\nu}(\beta), \alpha_{\nu+j+1}\right]
$$

with $\alpha_{\nu+j+1}>a_{\nu+1}(\beta)$. For fixed $b_{1}, \ldots, b_{j}$ this defines an interval $E(b)$ 
(suppressing $\nu, n, j)$ with endpoints $p_{j+\nu}(\alpha) / q_{j+\nu}(\alpha)$ and $p_{j+\nu+1}(\alpha) / q_{j+\nu+1}(\alpha)$ of length $1 / q_{j+\nu}(\alpha) q_{j+\nu+1}(\alpha)$. If $P_{i} / Q_{i}$ denotes the convergents of $\left[0, b_{1}, \ldots, b_{j}\right]$, then the set $F(b)$ of all $\alpha$ of the form $\left[0, b_{1}, \ldots, b_{j}, \alpha_{j+1}\right]$ is an interval with endpoints $P_{j} / Q_{j}$ and $\left(P_{j}+P_{j-1}\right) /\left(Q_{j}+Q_{j-1}\right)$ of length $1 / Q_{j}\left(Q_{j}+Q_{j-1}\right)$. Hence,

$$
|E(b)| /|F(b)| \leqslant Q_{j}^{2} / q_{j+\nu}(\alpha)^{2} .
$$

We use the well-known identity

$$
q_{j+\nu}(\alpha)=Q_{j} q_{\nu}(\beta)+Q_{j-1} q_{\nu-1}(\beta)
$$

to obtain

$$
|E(b)| /|F(b)| \leqslant 1 / q_{\nu}(\beta)^{2} .
$$

Now, where $\mathcal{U}_{b}$ means the union over all $1 \leqslant b_{1}, \ldots, b_{j}<\infty$, we have

$$
\bigcup_{b} E(b)=E_{\nu, n, j} \text { and } \bigcup_{b} F(b)=(0,1),
$$

where both unions are disjoint. Thus, we conclude that

$$
\left|E_{\nu, n, j}\right| \leqslant 1 / q_{\nu}(\beta)^{2}
$$

(we emphasize that this is independent of $j$ ).

Now, from the definition of $\rho_{\nu}(n, \alpha, \beta)$, if $\alpha \notin E_{\nu, n}$, where

$$
E_{\nu, n}=\bigcup_{j=n-\nu+1}^{n-1} E_{\nu, n, j},
$$

then $\rho_{\nu}(n, \alpha, \beta)=0$. Moreover, $\left|E_{\nu, n}\right| \leqslant \nu / q_{\nu}(\beta)^{2}$.

Set $l(n)=\left[n^{1 / 4}\right]$. Set

$$
E_{n}=\bigcup_{\nu \geqslant l(n)} E_{\nu, n}
$$

Since trivially $\rho_{\nu}(n, \alpha, \beta) \leqslant \nu$ for all $\nu, n$ we see that if $\alpha \notin E_{n}$

$$
\frac{1}{n} \sum_{\nu=0}^{\infty} \rho_{\nu}(n, \alpha, \beta) \leqslant \frac{1}{n} \sum_{\nu=0}^{l(n)-1} \nu \leqslant \frac{l(n)^{2}}{n} .
$$

Thus, we see that, if for any positive integer $m, \alpha \notin \bigcup_{n \geqslant m} E_{n}$, then

$$
\lim _{n \rightarrow \infty} \frac{1}{n} \sum_{\nu=0}^{\infty} \rho_{\nu}(n, \alpha, \beta)=0 .
$$

But the measure of the excluded set can be determined as

$$
\begin{aligned}
\left|\bigcap_{m=1}^{\infty} \bigcup_{n \geqslant m} E_{n}\right| & =\lim _{m \rightarrow \infty}\left|\bigcup_{n \geqslant m} E_{n}\right| \leqslant \lim _{m \rightarrow \infty} \sum_{n=m}^{\infty}\left|E_{n}\right| \\
& \leqslant \lim _{m \rightarrow \infty} \sum_{n=m}^{\infty} \frac{l(n)}{q_{l(n)}(\beta)^{2}}=0,
\end{aligned}
$$

since it is well known that $q_{\nu}(\beta) \geqslant 2^{(v-1) / 2}$; and so we see that $\Sigma_{n=2}^{\infty} l(n) / q_{l(n)}(\beta)^{2}$ is a convergent series. 
4. Comparison of the RCF and NICF. We will now give a very explicit algorithm for converting the RCF into the NICF. Theorem 3 will be an easy consequence of this procedure.

First, we will set up the notation and describe the NICF. For comparison we begin by briefly describing the familiar RCF (see [2]).

Let $\alpha$ be an irrational number. Let $a_{0}=[\alpha]$ denote the largest integer $\leqslant \alpha$. Let $\alpha_{0}=\alpha$ and, inductively, ${ }^{* *}$

$$
\alpha_{k}=\frac{1}{\alpha_{k-1}-a_{k-1}}, \quad a_{k}=\left[\alpha_{k}\right] .
$$

Then

$$
\alpha=\alpha_{0}=a_{0}+\frac{1}{a_{1}+\frac{1}{a_{2}+\frac{1}{a_{3}+\cdots}},}
$$

which we abbreviate as

$$
\alpha=\left[a_{0}, a_{1}, a_{2}, \ldots\right]
$$

Set $p_{-2}=0, q_{-2}=1, p_{-1}=1, q_{-1}=0$ and, inductively, for $k \geqslant 0, p_{k}=a_{k} p_{k-1}+$ $p_{k-2}, q_{k}=a_{k} q_{k-1}+q_{k-2}$. Then the $a_{k}$ are called the partial quotients of $\alpha$, and the $p_{k} / q_{k}$ are called the RCF convergents of $\alpha$. Moreover,

$$
p_{k} / q_{k}=\left[a_{0}, a_{1}, \ldots, a_{k}\right] \text { and } \alpha_{k}=\left[a_{k}, a_{k+1}, \ldots\right] \text {. }
$$

Now, for an irrational number $\alpha$ we will give the NICF algorithm (see [6], [7], [8]). Let $N(\alpha)$ denote the integer closest to $\alpha$. Set $\theta_{0}=\alpha, b_{0}=N(\alpha)$ and, inductively, set

$$
\theta_{n}=\frac{1}{b_{n-1}-\theta_{n-1}}, \quad b_{n}=N\left(\theta_{n}\right)
$$

Then

$$
\alpha=\theta_{0}=b_{0}-\frac{1}{b_{1}-\frac{1}{b_{2}-\cdots},}
$$

which we abbreviate as

$$
\alpha=\left(b_{0}, b_{1}, b_{2}, \ldots\right)
$$


Set $A_{-2}=0, B_{-2}=-1, A_{-1}=1, B_{-1}=0$ and, inductively, for $n \geqslant 0$,

$$
A_{n}=b_{n} A_{n-1}-A_{n-2}, \quad B_{n}=b_{n} B_{n-1}-B_{n-2} \text {. }
$$

The $A_{n} / B_{n}$ are called the NICF convergents of $\alpha$. Moreover,

$$
A_{n} / B_{n}=\left(b_{0} ; b_{1}, \ldots, b_{n}\right), \quad \theta_{n}=\left(b_{n} ; b_{n+1}, \ldots\right) .
$$

We now describe how, given the RCF, we can obtain the NICF from it.

THEOREM 4. For a given integer $n \geqslant-1$ there are 16 different possibilities for $b_{n}, A_{n}, B_{n}, A_{n-1}, B_{n-1}$ and $\theta_{n+1}$ given by the following table, where $k$ is chosen so that $A_{n}= \pm p_{k}, B_{n}= \pm q_{k}$ (same sign).

\begin{tabular}{lcccccccc}
\hline & I & II & III & IV & V & VI & VII & VIII \\
\hline$b_{n}$ & $a_{k}$ & $-a_{k}$ & $1+a_{k}$ & $-\left(1+a_{k}\right)$ & $1+a_{k-1}$ & $-\left(1+a_{k-1}\right)$ & $2+a_{k-1}$ & $-\left(2+a_{k-1}\right)$ \\
$A_{n}$ & $\pm p_{k}$ & $\pm p_{k}$ & $\pm p_{k}$ & $\pm p_{k}$ & $\pm p_{k}$ & $\pm p_{k}$ & $\pm p_{k}$ & $\pm p_{k}$ \\
$A_{n-1}$ & $\pm p_{k-1}$ & $\mp p_{k-1}$ & $\pm p_{k-1}$ & $\mp p_{k-1}$ & $\pm p_{k-2}$ & $\mp p_{k-2}$ & $\pm p_{k-2}$ & $\mp p_{k-2}$ \\
$\theta_{n+1}$ & $-\alpha_{k+1}$ & $\alpha_{k+1}$ & $-\alpha_{k+1}$ & $\alpha_{k+1}$ & $1+\alpha_{k+1}$ & $-\left(1+\alpha_{k+1}\right)$ & $1+\alpha_{k+1}$ & $-\left(1+\alpha_{k+1}\right)$ \\
\hline
\end{tabular}

The signs for $A_{n}, A_{n-1}$ are the respective signs given there, e.g. if in case IV, $A_{n}=$ $-p_{k}$, then $A_{n-1}=p_{k-1}$. To obtain $B_{n}$ use $q_{k}$ instead of $p_{k}$ with the same sign; and similarly for $B_{n-1}$. To begin use case II for $n=-1=k$ with the top choice of signs (ignore $b_{-1}$ and $a_{-1}$ ).

To obtain the table entry for $b_{n+1}, A_{n+1}, A_{n}$ and $\theta_{n+2}$, there are two cases. table).

Case a: $a_{k+2} \geqslant 2$. Here $A_{n+1} / B_{n+1}=p_{k+1} / q_{k+1}$ (i.e., $k \mapsto k+1$ in the table).

Case b: $a_{k+2}=1$. Here $A_{n+1} / B_{n+1}=p_{k+2} / q_{k+2}$ (i.e., $k \longmapsto k+2$ in the

The scheme below describes how to go from case to case as $n \mapsto n+1$ :
$\mathrm{I}^{a^{\mathrm{II}^{-}}} \mathrm{VI}^{-}$
II $\stackrel{a}{a}_{\mathrm{b}^{+}}^{\mathrm{I}^{+}}$
$\mathrm{III} \underset{b}{a_{\mathrm{VI}^{-}}}$
$\mathrm{IV}{\stackrel{b^{\prime}}{\mathrm{I}^{+}} \mathrm{V}^{+}}^{\mathrm{I}^{+}}$

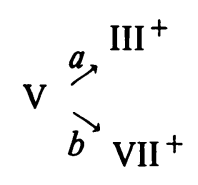
$\mathrm{VI}{\stackrel{\mathrm{J}^{\mathrm{I}}}{\mathrm{IVII}^{-}}}^{\mathrm{VII}^{-}}$

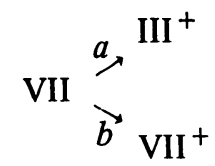

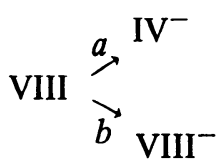

The "+", "-" superscripts indicate that either the choice of signs stays the same (for "+") or reverses (for "-").

Note finally that in cases I, II, III, IV, $a_{k+1} \geqslant 2$ and in cases V, VI, VII, VIII, $a_{k}=1$. 
Before proving Theorem 4, note that Theorem 3 is an immediate consequence. The proof given in [8] of Theorem 3 is much more direct but I think Theorem 4 has some independent interest. To aid in the understanding of the statement of Theorem 4 an example is worked out.

Let $\alpha=\sqrt{13 / 2}=[2 ; \overline{1,1,4}]$. For $n=k=-1, a_{k+1}=a_{1}=1$. So II $\rightarrow$ $\mathrm{V}^{+}, n=0, k=1$ and $b_{0}=1+a_{k-1}=1+a_{0}=3$. Now $a_{k+2}=a_{3}=4$ so $\mathrm{V} \rightarrow$ $\mathrm{III}^{+}, n=1, k=2$ and $b_{1}=1+a_{k}=1+a_{2}=2$. Again, $a_{k+2}=a_{4}=1$ so III $\rightarrow$ $\mathrm{VI}^{-}, n=2, k=4$ and $b_{2}=-\left(1+a_{3}\right)=-5$. Once again, $a_{k+2}=a_{6}=4$ so $\mathrm{VI} \rightarrow$ $\mathrm{IV}^{-}, n=3, k=5$ and $b_{3}=-\left(1+a_{5}\right)=-2$. Finally, $a_{k+2}=a_{7}=1$ so $\mathrm{IV}^{-} \rightarrow$ $\mathrm{V}^{+}, n=4, k=7$, so $b_{4}=1+a_{6}=5$ and the cycle is complete. Hence, $\sqrt{13 / 2}=$ $(3 ; \overline{2,-5,-2,5})$. (Note that the period is in fact longer here, contrary to statements made in [7], [8]. However, this in no way affects anything done in those papers.)

The proof of Theorem 4 using induction on $n$ is simply to verify each of the cases. We will only do this for

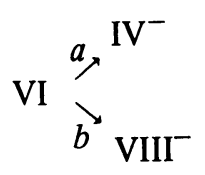

the other cases being similar. First assume that $a_{k+2} \geqslant 2$ (Case a). Then, since $\theta_{n+1}$ $=-\left(1+\alpha_{k+1}\right)$, we see that $b_{n+1}=N\left(\theta_{n+1}\right)=-\left(1+a_{k+1}\right) ; A_{n+1}=b_{n+1} A_{n}-$ $A_{n-1}=-\left(1+a_{k+1}\right)\left( \pm p_{k}\right)-\left(\mp p_{k-2}\right)=\mp p_{k+1} \mp\left(p_{k}-p_{k-1}-p_{k-2}\right)=\mp p_{k+1}$ since $a_{k}=1 ; A_{n}= \pm p_{k} ; \theta_{n+2}=1 /\left(b_{n+1}-\theta_{n+1}\right)=1 /\left(-a_{k+1}+\alpha_{k+1}\right)=\alpha_{k+2}$; thus, we have $\mathrm{IV}^{-}$. Now assume $a_{k+2}=1$ (Case b). Then again $\theta_{n+1}=$ $-\left(1+\alpha_{k+1}\right)$ implies $b_{n+1}=N\left(\theta_{n+1}\right)=-\left(2+a_{k+1}\right) ; A_{n+1}=-\left(2+a_{k+1}\right)\left( \pm p_{k}\right)$ $-\left(\mp p_{k-2}\right)=\mp\left(p_{k+1}+p_{k}\right) \mp\left(p_{k}-p_{k-1}-p_{k-2}\right)=\mp p_{k+2}$ since $a_{k}=a_{k+2}=1$; $A_{n}= \pm p_{k} ; \theta_{n+2}=1 /\left(-1-a_{k+1}+\alpha_{k+1}\right)=1 /\left(-1+\alpha_{k+2}^{-1}\right)=-\alpha_{k+2} /\left(\alpha_{k+2}-1\right)$ $=-\left(1+\alpha_{k+3}\right)$, since $a_{k+2}=1$; thus, we have VIII ${ }^{-}$.

5. Proof of Theorem 1. Theorem 2 will now be applied to prove Theorem 1 . Recall that given $\alpha=\left[a_{0} ; a_{1}, a_{2}, \ldots\right]$ we have for integers $n \geqslant-1, k(n)$ defined so that $A_{n} / B_{n}=p_{k(n)} / q_{k(n)}$. From Theorem 3, we have the following recursion for $k(n)$ :

$$
\begin{aligned}
k(-1) & =-1 \\
k(n+1) & =\left\{\begin{array}{l}
k(n)+1 \text { if } a_{k(n)+2} \geqslant 2 \\
k(n)+2 \text { if } a_{k(n)+2}=1
\end{array}\right\} .
\end{aligned}
$$

Define $\Phi_{\nu}(n, \alpha)$ for integers $\nu \geqslant 0, n \geqslant-1$ to be the number of integers $j$ satisfying $0 \leqslant j \leqslant k(n)-\nu$ such that $a_{j+1}=a_{j+2}=\cdots=a_{j+\nu}=1$. That is, $\Phi_{\nu}(n, \alpha)$ is the number of strings of $\nu$ consecutive 1's in the first $k(n)$ partial quotients ( $a_{0}$ is excluded). Also, $\Phi_{\nu}(n, \alpha)=\varphi_{\nu}(k(n), \alpha,(\sqrt{5}-1) / 2)$. The relation between the NICF and the frequency of digits in the RCF is given by

LEMMA 5. $k(n)=n+\sum_{\nu=1}^{\infty}(-1)^{\nu-1} \Phi_{\nu}(n, \alpha)$. 
Proof. Denoting the right-hand side by $h(n)$, it suffices to show that $h(n)$ satisfies the same recursion (5) that $k(n)$ does. First, it is clear that $\Phi_{\nu}(-1, \alpha)=0$ for all $\nu \geqslant 1$ and so $h(-1)=-1$ as desired. Set

$$
\epsilon_{n}= \begin{cases}0 & \text { if } a_{k(n)+2} \geqslant 2 \\ 1 & \text { if } a_{k(n)+2}=1\end{cases}
$$

so that $k(n+1)=k(n)+1+\epsilon_{n}$. Then, from (5) with $h(n)$ we see that we need to prove that for all $n \geqslant-1$

$$
n+1+\sum_{\nu=1}^{\infty}(-1)^{\nu-1} \Phi_{\nu}(n+1, \alpha)=n+\sum_{\nu=1}^{\infty}(-1)^{\nu-1} \Phi_{\nu}(n, \alpha)+1+\epsilon_{n}
$$

or

$$
\sum_{\nu=1}^{\infty}(-1)^{\nu-1} \Phi_{\nu}(n+1, \alpha)=\sum_{\nu=1}^{\infty}(-1)^{\nu-1} \Phi_{\nu}(n, \alpha)+\epsilon_{n}
$$

There are two cases.

Case a: $k(n)=k(n-1)+1$. That is, $a_{k(n-1)+2} \geqslant 2$ or $a_{k(n)+1} \geqslant 2$. We see then that $\Phi_{1}(n+1, \alpha)=\Phi_{1}(n, \alpha)+\epsilon_{n}$ and $\Phi_{\nu}(n+1, \alpha)=\Phi_{\nu}(n, \alpha)$ for all $\nu \geqslant 2$, and it is clear that (6) holds in this case.

Case b: $k(n)=k(n-1)+2$. That is, $a_{k(n)}=a_{k(n-1)+2}=1$. Now if $a_{k(n)+1} \geqslant 2$, then as before $\Phi_{1}(n+1, \alpha)=\Phi_{1}(n, \alpha)+\epsilon_{n}$ and $\Phi_{\nu}(n+1, \alpha)=$ $\Phi_{\nu}(n, \alpha)$ for all $\nu \geqslant 2$; and we are done. So assume $a_{k(n)+1}=1$. Choose $r \geqslant 0$ so that $a_{r} \neq 1, a_{r+1}=a_{r+2}=\cdots=a_{k(n)+1}=1$ (if $a_{1}=\cdots=a_{k(n)+1}=1$ set $r=$ $0)$. Then, we see easily that

$$
\Phi_{\nu}(n+1, \alpha)= \begin{cases}\Phi_{\nu}(n, \alpha)+1+\epsilon_{n} & \text { if } 1 \leqslant \nu \leqslant k(n)+1-r \\ \Phi_{\nu}(n, \alpha)+\epsilon_{n} & \text { if } \nu=k(n)+2-r \\ \Phi_{\nu}(n, \alpha) & \text { if } \nu>k(n)+2-r .\end{cases}
$$

Now we observe that $k(n)+1-r$ is even. Since $a_{r} \geqslant 2$ (or $r=0$ ) and $a_{r+1}=1$, we see that there is an $m$ such that $r+1=k(m)$. Then $a_{k(m)}=a_{k(m)+1}=\cdots=$ $a_{k(n)}=1$ and so we see $k(n)-k(m)$ is even. Thus, $k(n)+1-r=k(n)+2-k(m)$ is even, also. From this and (7) we see that the first $k(n)+1-r$ terms of $1+\epsilon_{n}$ in (6) cancel out, and the $\epsilon_{n}$ in the term for the odd number $k(n)+2-r$ is exactly what is needed. This completes the proof of Lemma 5.

Theorem 1 now follows, since for almost all $\alpha$

$$
\begin{aligned}
\lim _{n \rightarrow \infty} \frac{n}{k(n)} & =\lim _{n \rightarrow \infty} \frac{1}{k(n)}\left(k(n)+\sum_{\nu=1}^{\infty}(-1)^{\nu} \Phi_{\nu}(n, \alpha)\right) \\
& =\lim _{n \rightarrow \infty} \frac{1}{k(n)} \sum_{\nu=0}^{\infty}(-1)^{\nu} \varphi_{\nu}\left(k(n), \alpha, \frac{\sqrt{5}-1}{2}\right)=\frac{\log \left(\frac{1+\sqrt{5}}{2}\right)}{\log 2}
\end{aligned}
$$

from Lemma 5 and Theorem 2(b). 
Department of Mathematics

University of Maryland

College Park, Maryland 20742

1. P. BILlingSLEY, Ergodic Theory and Information, Wiley, New York, 1965.

2. A. KHINTCHINE, Continued Fractions, Univ. of Chicago Press, Chicago, 1964.

3. W. PHILIPP, "Some metrical theorems in number theory," Pacific J. Math., v. 29, 1967, pp. 109-127.

4. D. SHANKS, Review of the UMT file: "Two related quadratic surds having continued fractions with exceptionally long periods," Math. Comp., v. 28, 1974, pp. 333-334.

5. J. SCHOCKLEY, Introduction to Number Theory, Holt, Rinehart and Winston, New York, 1967.

6. H. WILLIAMS \& J. BROERE, "A computational technique for evaluating $L(1, \chi)$ and the class number of a real quadratic field," Math. Comp., v. 30, 1976, pp. 887-893.

7. H. WILLIAMS \& P. BUHR, "Calculation of the regulator of $Q(\sqrt{d})$ by use of the nearest integer continued fraction algorithm," Math. Comp., v. 33, 1979, pp. 369-381.

8. H. WILLIAMS, "Some results concerning the nearest integer continued fraction algorithm," J. Reine Angew. Math. (To appear.)

9. G. J. RIEGER, “Über die mittlere Schrittanzahl bei Divisionsalgorithmen," Math. Nachr., v. 82, 1978, pp. $157-180$. 